\title{
Synchronous metastasis from double primary cancers in a single left supraclavicular lymph node
}

\author{
Ye An Kim ${ }^{1,2}$, Hye Sook Min³, Sun Wook Cho ${ }^{4}$, and Young Joo Park ${ }^{1,4}$
}

\begin{abstract}
${ }^{1}$ Department of Internal Medicine, Seoul National University College of Medicine, Seoul; ${ }^{2}$ Department of Internal Medicine, Veterans Health Service Medical Center, Seoul; ${ }^{3}$ Department of Preventive Medicine, Graduate School of Public Health, Seoul National University, Seoul; ${ }^{4}$ Department of Internal Medicine, Seoul National University Hospital, Seoul, Korea
\end{abstract}

Received: August 22, 2016 Revised : September 6, 2016 Accepted: October 9, 2016

\section{Correspondence to}

Young Joo Park, M.D.

Tel: +82-2-2072-4183

Fax: +82-2-762-2199

E-mail: yjparkmd@snu.ac.kr
A 39-year-old woman was referred to our hospital for invasive cervical cancer. On initial physical examination, inguinal lymph nodes, but not supraclavicular lymph nodes, were palpable. Positron emission tomography revealed multiple bone metastasis with hypermetabolic lymph nodes in the abdominal and pelvic cavity, left supraclavicular fossa, and a mild hypermetabolic nodular lesion in the left thyroid (Fig. 1A). Gun biopsy was performed for the left supraclavicular lymph node. Ultrasonography indicated the left thyroid nodule was a $1 \mathrm{~cm}$ sized hypoechoic lesion with inner calcification (Fig. 1B). Papillary thyroid carcinoma was diag- nosed with fine needle aspiration (Fig. ${ }_{1 C}$ ). A lymph node specimen revealed synchronous metastasis from a double primary origin (Fig. 2A, ×40). One metastatic lesion (Fig. 2B, blue square, $\times 100$ ) showed papillary growth and was positive for thyroglobulin immunohistochemical stain (Fig. 2C, $\times 100$ ), consistent with thyroid carcinoma. Another metastatic lesion (Fig. 2D, yellow square, $\times 100$ ) was negative for thyroglobulin (Fig. 2E, ×100). Immunohistochemical staining was positive for $\mathrm{p} 16$ (Fig. 2F, ×100) and p63 (Fig. 2G, ×100), verifying metastatic squamous cell carcinoma. Final diagnosis was advanced cervical cancer (stage IVb), and she re-

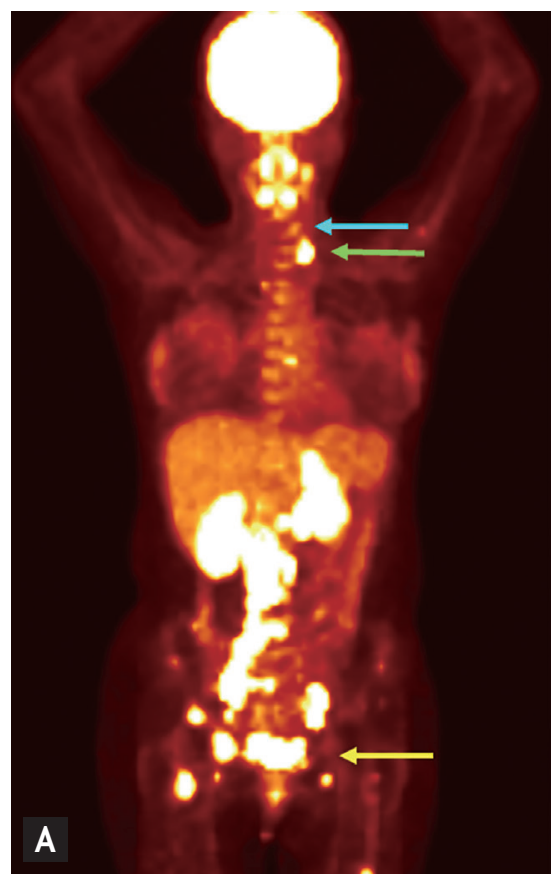

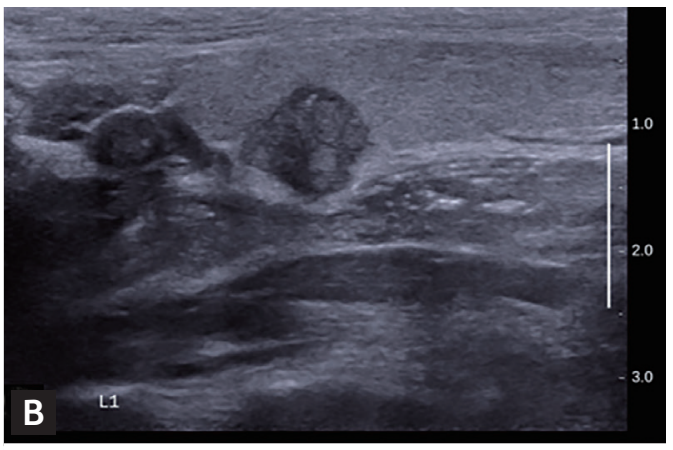

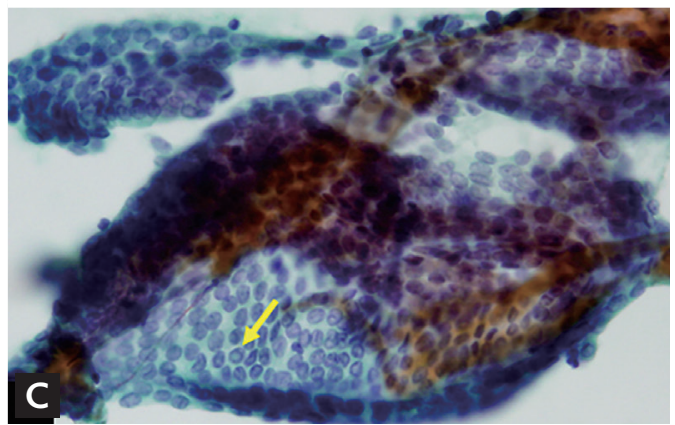

Figure 1. (A) Positron-emission and computed tomography scans revealed multiple bone metastasis including pelvic bone, sacrum, and lumbar spine. Multiple conglomerated lymph nodes in the abdominal and pelvic cavity (yellow arrow) and left supraclavicular fossa (green arrow) were also hypermetabolic with mild hypermetabolic nodular lesion in the left thyroid (blue arrow). (B) The $1 \mathrm{~cm}$ left thyroid nodule was a well demarcated hypoechoic lesion with inner calcification on neck ultrasonography. (C) Cytology specimen revealed papillary thyroid carcinoma (May Grumwald Giemsa, ×400). Yellow arrow indicates pseudoinclusion body in the nucleus. 

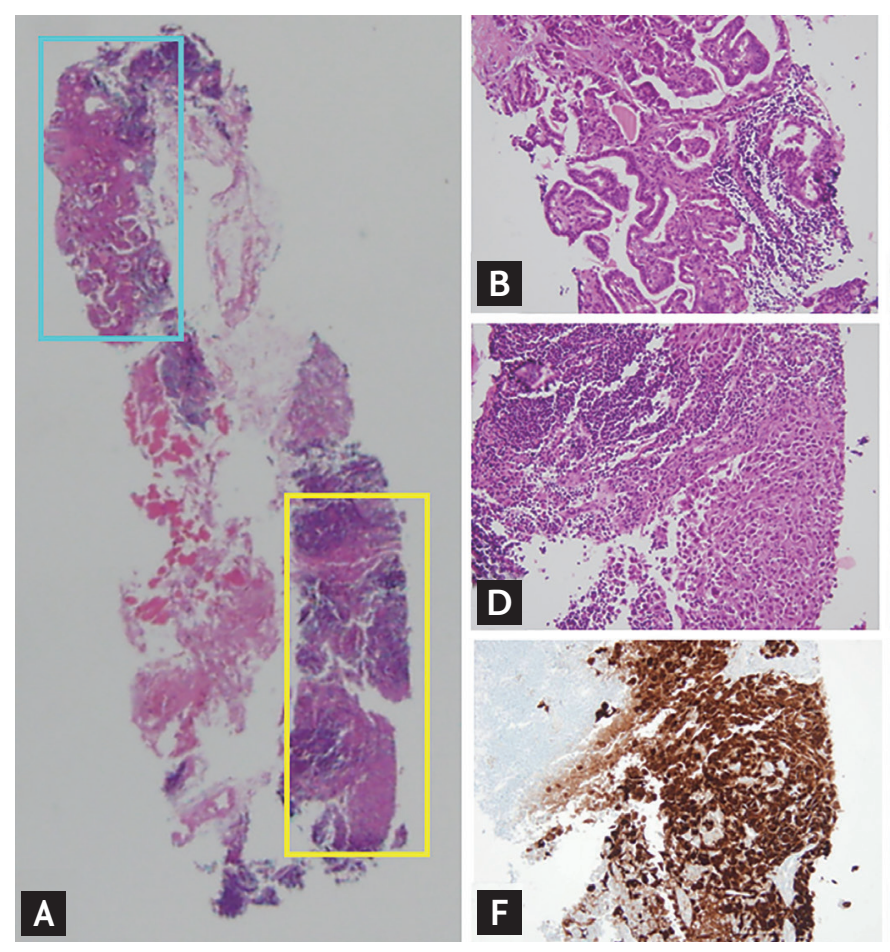

C

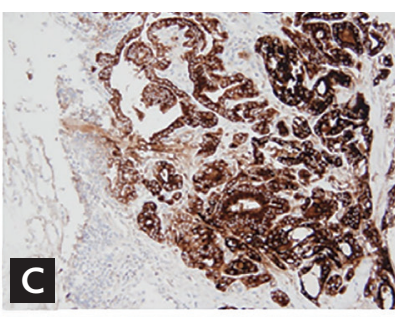

Figure 2. (A) Lymph node specimen presents two metastatic lesions marked with blue and yellow squares $(\mathrm{H} \& \mathrm{E}, \times 40)$. One metastatic lesion (blue square) showed papillary growth (B, $\mathrm{H} \& \mathrm{E}, \times 100)$ and was positive for thyroglobulin immunohistochemical stain $(\mathrm{C}, \times 100)$. Another metastatic lesion (yellow square) showed squamous cell carcinoma feature (D, H\&E, $\times 100)$ and was negative for thyroglobulin $(\mathrm{E}, \times 100)$. Immunohistochemical staining verified metastatic squamous cell carcinoma from uterine cervix, which was positive for $\mathrm{p} 16(\mathrm{~F}$, G

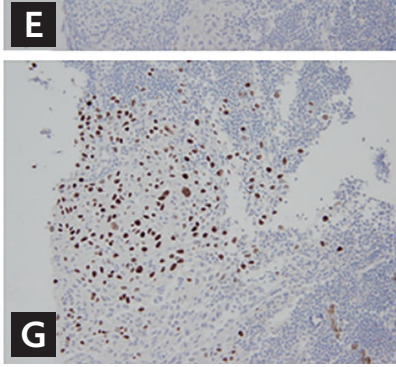
$\times 100)$ and p63 $(\mathrm{G}, \times 100)$. ceived palliative chemotherapy. For thyroid cancer, low dose thyroxine was given for 3 months after confirming normal thyroid function and lost to follow-up.

The presence of double primary tumors is extremely rare. This is, to our knowledge, the first report of synchronous metastatic tumors in a single lymph node. The left supraclavicular lymph node, also known as Virchow's node, is located in the confluence of lymphatic drainage from abdominal and pelvic cavities. Metastases from the gastrointestinal tract are occasionally present ( $5 \%$ to $8 \%$ for cervical cancer). Head and neck, lung, and breast cancers may also metastasize to the supraclavicular lymph nodes with no difference in incidence reported between left and right nodes. Most supraclavicular lymph node metastasis is related to a poor prognosis. For treatment plan development, life expectancies related to the primary malignancies should consider the patients' condition.

\section{Conflict of interest}

No potential conflict of interest relevant to this article was reported. 